\title{
EL ASEGURAMIENTO DE LA CALIDAD EN LAS PYMES ECUATORIANAS
}

\section{QUALITY ASSURANCE IN ECUADORIAN SMES}

\section{Teodoro Mauricio Escalante Bourne', Clarkent Rubén Mackay Castro², Teodoro Antonio Escalante Ramírez ${ }^{3}$, Rubén Alberto Mackay Véliz ${ }^{4}$}

\author{
1,2,3 - Universidad de Guayaquil, Guayaquil, Ecuador \\ 4 - Instituto Técnico Bolivariano, Guayaquil, Ecuador
}

\begin{abstract}
1. Email: teodoro.escalantebo@ug.edu.ec ORCID: $\underline{\text { https://orcid.org/0000-0001-9351-4286 }}$
2. Email: ruben.mackayc@ug.edu.ec ORCID: $\underline{\text { https://orcid.org/0000-0002-2657-2168 }}$

3. Email: teodoro.escalanter@ug.edu.ec ORCID: https://orcid.org/0000-0002-3682-3098
\end{abstract}

4. Email: rmackay@itb.edu.ec ORCID: https://orcid.org/0000-0001-9272-3991

Recibido: 11/05/2021 Aceptado: 21/07/2021

Para Citar: Escalante Bourne, T. M., Mackay Castro, C. R., Escalante Ramírez, T. A., \& Mackay Véliz, R. A. (2021). El aseguramiento de la calidad en las PYMES ecuatorianas. Revista Publicando, 8(31), 71-81. https://doi.org/10.51528/rp.vol8.id2235

\begin{abstract}
Resumen:
Una empresa puede estar clasificada como grande, mediana o pequeña dependiendo de su tamaño organizacional y volumen de operaciones. Al conjunto de empresas pequeñas y medianas se les conoce como PYMES. Por lo general, se encuentran presentes en todos los sectores de la actividad económica ecuatoriana, con mayor presencia en el sector comercio, encontrándose en un mundo de emprendedores donde la modernización y globalización juegan un papel preponderante para alcanzar el aseguramiento de la calidad. Es por ello que el objetivo general de esta investigación se centra en analizar la importancia del aseguramiento de la calidad en las PYMES de Ecuador; encontrando como resultado que éstas ejercen una fuerte influencia sobre el crecimiento económico del país al ser una elevada fuente generadora de empleo, que permite contribuir a la mejora social, económica y financiera para combatir los índices de pobreza y generar cambios en las políticas de productividad nacional. Por otro lado, la implementación de sistemas de gestión de calidad como las Normas ISO en las PYMES, permite que este tipo de organización se modernice y organice en su estructura interna y de procesos, para poder elevar los estándares de calidad en los productos o servicios ofrecidos al analizar y comprender las necesidades, posibles expectativas y satisfaciendo los requerimientos de los clientes; lo que conlleva a conseguir el aseguramiento de la calidad brindando la oportunidad de expandirse a nuevos mercados como el europeo donde existe un alto índice de competitividad y exigencia.
\end{abstract}

Palabras clave: pymes, desarrollo, sistemas de gestión, aseguramiento de la calidad, competitividad.

\begin{abstract}
:
A company may be classified as large, medium or small, depending on its organizational size and volume of operations. All small and mediumsized companies are known as SMEs. In general, they are present in all sectors of the Ecuadorian economic activity, with greater presence in the commerce sector, being in a world of entrepreneurs where modernization and globalization play a preponderant role to achieve quality assurance. That is why the general objective of this research focuses on analyzing the importance of quality assurance in SMEs in Ecuador; finding as a result that they exert a strong influence on the country's economic growth as they are a high source of employment generation, which contributes to social, economic and financial improvement to combat poverty rates and generate changes in national productivity policies. On the other hand, the implementation of quality management systems such as ISO Standards in SMEs, allows this type of organization to modernize and organize its internal structure and processes, in order to raise the quality standards in the products or services offered by analyzing and understanding the needs, possible expectations and satisfying customer requirements; which leads to achieve quality assurance providing the opportunity to expand into new markets such as Europe where there is a high rate of competitiveness and demand.
\end{abstract}

Keywords: SMEs, development, management systems, quality assurance, competitiveness. 


\section{INTRODUCCIÓN}

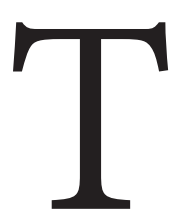

radicionalmente, la economía mundial se mueve a través del sector empresarial. Una empresa puede estar clasificada como grande, mediana o pequeña dependiendo de su tamaño organizacional y volumen de operaciones. Al conjunto de empresas pequeñas y medianas se les conoce como PYMES y el número de éstas en cualquier país es normalmente elevado, representando aproximadamente más del $80 \%$ de los negocios de una economía.

En este sentido, la economía del Ecuador es fortalecida por la PYMES, estas juegan un papel muy importante ya que son las responsables de generar miles de plazas de empleo y dinamizar el capital del país. Por lo general, se encuentran presentes en todos los sectores de la actividad económica, con mayor presencia en el sector comercio, enfrentándose a un mundo de emprendedores y siendo de gran importancia no solo por las aportaciones a la producción nacional sino también por la flexibilidad de adaptación a nuevos rumbos; donde deben probar sus capacidades en la adopción de alianzas estratégicas que generen un cambio ya sea de tipo tecnológico, social o de generación de empleo (Delgado y Chávez, 2018).

En el mismo orden de ideas, los procesos de cualquier organización siempre están en constante movimiento y es por ello que deben aplicar la modernización para estar en mejora continua a pesar de que ya se encuentren en un mercado estable durante años. Con esto lograran mejorar el rendimiento, el cumplimiento de metas planteadas e innovar para posicionarse de mejor manera en el mercado, de tal forma que alcancen los requerimientos exigidos por sus clientes (Terán y Alvarado, 2016).

Según la Universidad Cooperativa de Colombia (2018) esto se logra a través de los Sistemas de Gestión de Calidad; los cuales no son más que una herramienta que permite a cualquier organización planear, ejecutar y controlar las actividades necesarias para el desarrollo de la misión, a través de la prestación de servicios con altos estándares de calidad. Estos sistemas se volvieron muy populares alrededor del mundo luego de la segunda Guerra Mundial, trayendo consigo una mayor exigencia en todo sentido a las compañías para que estas pudieran mantenerse o ingresar al mercado mundial; lo que trajo como consecuencia la aparición de un conjunto de normas internacionales para alcanzar la estandarización. Entre las más conocidas y aplicadas se encuentran las normas ISO.

Según Palacio et al. (2018), las normas ISO son un conjunto de normas orientadas a ordenar la gestión de una empresa en sus distintos ámbitos para lograr una adecuada gestión de calidad que permita cumplir con los estándares mundiales obteniendo así el aseguramiento de la calidad de los productos.

Es aquí donde la gestión empresarial de dichas organizaciones cobra relevancia para su crecimiento, desarrollo y sostenibilidad en el tiempo. La participación de la alta dirección permitirá generar estrategias, coordinar recursos (equipos, personas, procesos, entre otros) para adaptarse a esta nueva visión empresarial, lo que las ayudara a sostenerse y mantenerse en los mercados por largos periodos de tiempo 
ajustándose a las demandas del mercado actual (Palacio et al. 2018).

Todo esto incide en la necesidad de implementar sistemas de gestión que permitan asegurar la calidad de sus bienes, productos o servicios; lo que conlleva a que el aseguramiento de la calidad pase a ser una tarea obligatoria en el contexto moderno (sin importar la actividad económica o el tamaño de las organización), donde la obtención de mejores resultados se vuelve una prioridad para poder ser más competitivo y cumplir con las exigencias actuales de la globalización (Pereira, 2019).

En la Norma ISO 9000:2005, el aseguramiento de la calidad se define como un conjunto de actividades coordinadas para dirigir y controlar una organización, orientada a proporcionar confianza en que se cumplirán los requisitos de la calidad. En tal sentido, el aseguramiento de la calidad está dado por acciones planificadas y sistemáticas, necesarias para proporcionar la confianza adecuada de que un producto o servicio satisfará los requerimientos dados sobre calidad. Esta herramienta gerencial está orientada y enfocada en un proceso pro activo de calidad, con el fin de mejorar las técnicas de desarrollo a través del manejo de la calidad y por medio de evaluaciones de adecuación proporcionadas por auditorías periódicas de las operaciones de todo el sistema.

Ahora bien, según diversos estudios en las PYMES ecuatorianas el factor más común para la desaparición de este tipo de organización se debe principalmente a que se estancan como empresas empíricas donde prevalece la inexistencia de un sistema de gestión y por tanto no existe el aseguramiento de la calidad de los productos, bienes o servicios ofrecidos y es por esto que el objetivo general de esta investigación se centra en analizar la importancia del aseguramiento de la calidad en las PYMES de este país.

\section{METODOLOGÍA}

La metodología empleada es de diseño bibliográfico de tipo documental. El diseño se fundamenta en la revisión sistemática, rigurosa y profunda de material documental de cualquier clase, donde se efectúa un proceso de abstracción científica, generalizando sobre la base de lo fundamental, partiendo de forma ordenada y con objetivos precisos (Palella Stracuzzi \& Martins Pestana, 2010).

La investigación documental se concreta exclusivamente en la recopilación de información de diversas fuentes, con el objeto de organizarla describirla e interpretarla de acuerdo con ciertos procedimientos que garanticen confiabilidad y objetividad en la presentación de los resultados (Palella Stracuzzi \& Martins Pestana, 2010). La composición de este artículo se creó a partir de una descripción general de la documentación especial sobre este tema, teniendo en cuenta las publicaciones de revistas indexadas y libros actuales relacionados.

\section{RESULTADOS Y DISCUSIÓN}

PYME es el acrónimo de pequeña y mediana empresa. Se trata de la empresa mercantil, industrial o de otro tipo que tiene un número reducido de trabajadores y que registra ingresos moderados" (Rodríguez y Lesmes, 2019). Las pequeñas y medianas empresas de cualquier sector económico que comercializan bienes o servicios para generar rentabilidad. 
Las PYMES juegan un rol preponderante en la dinamización de la economía. Ventocilla (2014, Citado por Peña y Vega, 2018), afirma que "Las PYMES se vuelven sistemas creadores de valor económico cuando desarrollan dinámicas productivas y competitivas que les permiten enriquecer su contexto conectando a las personas con los mercados, en los cuales se encuentran los recursos requeridos para satisfacer sus necesidades, a la vez que se enriquecen en el proceso".

Este tipo de organizaciones se caracterizan por ser empresas de tipo familiar, las cuales, poseen capital bajo e infraestructura moderada y se han originado por capital inicial propio en su mayoría, de tal forma que se han convertido en una herramienta eficaz para combatir la pobreza $y$ forjar el pleno empleo, con la finalidad de que se pueda plasmar un verdadero desarrollo nacional (Delgado y Chávez, 2018).

Es por ello que, Pereira (2019) hace mención de Gávez y García (2012), pues estos autores consideran a las PYMES como un tema relevante en la actualidad económica, que genera interés en la creación y fortalecimiento de empresas cada vez competitivas como motor de desarrollo de sus administraciones. Dicho interés se refleja en el incremento porcentual que de manera considerable ha tenido el empleo, la producción y el comercio gracias al desarrollo de éstas a nivel mundial.

De la misma manera, Delgado y Chávez (2018) menciona que, de acuerdo a la base de datos de Ecuador denominada REDATAM del Instituto Nacional de Estadísticas y Censos (INEC), se indica que 99 de cada 100 establecimientos se catalogan como pequeña o mediana empresa. y explica que la fuente para el movimiento de la economía en Ecuador son las Pequeñas y medias empresas (PYMES) y gracias a ello existe un sin número de emprendimientos que se dan en el país, generando fuentes de trabajo y siendo el motor para que la economía gire constantemente. En el mismo orden de ideas, estos autores manifiestan que las Medianas y Pequeñas empresas en el Ecuador se dedican a la producción de bienes y servicios, generando empleo aportando al desarrollo social, produciendo, comprando y demandando productos con valor añadido.

Por otra parte, La Secretaria Nacional de Planificación, 2017 para abordar este tema en su Plan Nacional para el buen vivir 20172021 refiere entre sus objetivos: promover la productividad, competitividad y calidad de los productos nacionales, como también la disponibilidad de servicios conexos y otros insumos, para generar valor agregado y procesos de industrialización en los sectores productivos con enfoque a satisfacer la demanda nacional y de exportación. Esto ha logrado que las PYMES nacionales comiencen a pensar en los sistemas de gestión, dejando de lado el empirismo, el miedo a lo desconocido y arriesgándose al cambio en sus estilos de organización para comenzar a proyectarse de manera internacional y poder aumentar su competitividad.

Según Mendoza et al. (2018), el sistema de gestión de calidad corresponde a diversas acciones que afectan de forma positiva a toda la empresa mejorando continuamente la organización, procedimientos, procesos, recursos financieros, económicos y humanos, lo que vuelve más competitiva a las organizaciones que implementan estos sistemas. 
Ahora bien, según reflejan Palacio et al. (2018) en su trabajo titulado: Utilización de controles, auditorías administrativas y sistemas de gestión de la calidad en las PYMES; solo el 52,4\% de las empresas PYMES ecuatorianas se encuentran utilizando un sistema de gestión de la calidad. El sector industrial y productivo es quien más aplica estos sistemas, con el 61\%, y mantiene una cierta distancia de los sectores comercial y de servicios, cuyos porcentajes son de 53\% y $49 \%$ respectivamente. También concluyeron que la mayoría de estas organizaciones aplican las normas ISO $9000(59,60 \%)$ y el restante de ellas $(38,40 \%)$, utilizan otros sistemas para el aseguramiento de la calidad.

En tal sentido, a pesar de que los sistemas de gestión en las PYMES ecuatorianas han ido incrementándose en bajos porcentajes; esto denota que la necesidad de satisfacer al cliente y mejorar su competitividad, gestionar mediante la planeación, organización, dirección y control, han sido imperantes para lograr alcanzar asegurar la calidad y poder optimizar de una mejor manera los recursos con los que se cuenta con el objetivo de crecer, desarrollarse y mantenerse en el mercado así como también para expandirse en nuevos campos.

Como ya se mencionó con anterioridad, entre los métodos de gestión más utilizados para conseguir el aseguramiento de la calidad en las PYMES ecuatorianas se encuentran los sistemas ISO. Éstos están basados en un conjunto de normas establecidas por la Organización Internacional para la Estandarización y son un conjunto de directrices para el comercio, manufactura y comunicación (aplicadas a las pequeñas, medianas y grandes empresas) que permiten mejorar la calidad de sus productos o servicios al optimizar y controlar sus procesos. La implementación de las normas ISO genera una serie de ventajas para las pequeñas y medianas empresas y según Anexia Consultoria (2018) se muestran en la Tabla 1.

Tabla 1

Ventajas de la implementación de la Normas ISO en las PYMES

\section{VENTAJAS}

Demuestra el compromiso, responsabilidad e interés de implementar mejoras continuas en la empresa, lo que aporta seguridad a posibles accionistas, socios, vendedores de materia prima y clientes.

Mejora el rendimiento en las actividades, aplicaciones comerciales, ritmo de producción y resultados

Mejora la reputación a nivel mundial ya que aumenta la confianza que inspira la empresa.

Garantiza la satisfacción al cliente al escuchar sus requerimientos con más detalle.

Incentiva el crecimiento ya que tienen mejores tasas de ventas, supervivencia y un notable crecimiento del personal. 
Brinda mejoras continuas al ser capaz la organización de controlar y mejorar su rendimiento para alcanzar la eficacia en todos los aspectos.

Genera ventaja competitiva respecto a las otras empresas del sector que no cuentan con esta normativa.

Ahorra en recursos al mejorar el aprovechamiento de éstos y reducir los costos de producción, al mismo tiempo que aumenta la calidad del producto. De esta forma se reduce el retrabajo, el tiempo muerto, los accidentes laborales entre otros gastos innecesarios y empleados infelices.

Fuente: (Anexia Consultoria, 2018).

Debido al constante cambio que vive el mercado y la globalización, las normas ISO permiten a las empresas competir con otras más grandes equiparándose en eficiencia, para poder cumplir con los clientes que exigen proveedores con certificación de calidad y generando confianza en ellos, gracias a las mejoras en la eficacia y la productividad del producto o servicio, lo que brinda un valor añadido a la organización (Universitat Oberta de Catalunya, 2014).

Para que una PYME se vea beneficiada a nivel sistemático mediante un sistema de gestión, es necesario que se enfoque principalmente en sus propias características para poder establecer los procesos necesarios y documentarlos de tal forma que se pueda tener un flujo de proceso adecuado que permita utilizar los recursos correctamente e implantar los métodos y estrategias que permitirán la mejora y el logro de la calidad de su producto o servicio (Mendoza et al. 2018).

Tal como se menciona en la Norma ISO 9000 (2005), La Figura 1 ilustra el sistema de gestión de la calidad basado en procesos descrito en la familia de Normas ISO9000. Esta ilustración muestra que las partes interesadas juegan un papel significativo para proporcionar elementos de entrada a la organización. El seguimiento de la satisfacción de las partes interesadas requiere la evaluación de la información relativa a su percepción de hasta qué punto se han cumplido sus necesidades $y$ expectativas.

De esta forma, la gestión empresarial de dichas organizaciones cobra relevancia para su crecimiento, desarrollo y sostenibilidad, al basarse en métodos de gestión que permitan un enfoque sistemático e integrador de todas sus partes y que sea suficientemente satisfactorio para todos ejes involucrados. Entonces, la gestión empresarial o alta gerencia así como el trabajo en equipo juegan un papel preponderante al momento de implementar cualquier sistema ya que juntas lograran el crecimiento, desarrollo y sostenibilidad de toda la organización.

En tal sentido, abordar la gestión empresarial se constituye en una tarea obligatoria en el contexto moderno de los negocios sin importar la actividad económica o el tamaño de las organizaciones, máxime con las exigencias actuales de la globalización y la obtención de resultados como prioridad en cualquier empresa que se emprenda. Bajo esta óptica, la gestión organizacional se constituye en el eje de desarrollo, productividad y competitividad de las empresas del nuevo siglo, orientadas a la globalización de negocios y 
Figura 1

Modelo de un sistema de gestión de la calidad basado en procesos.
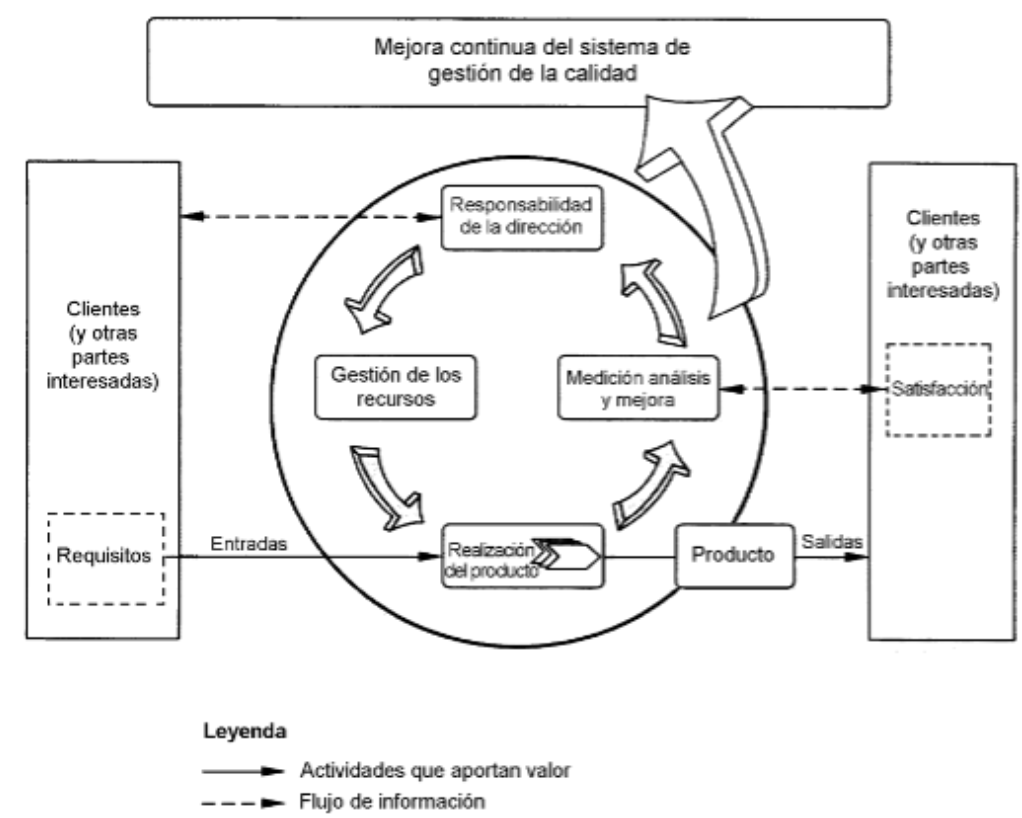

Fuente: (ISO 9000, 2005).

posicionamiento en los mercados (Pereira, 2019).

Ahora bien, según la Norma ISO 9000 (2005), el aseguramiento de calidad ha sido englobado en la gestión de la calidad, proporcionando un modelo que establece una serie de requisitos al propio sistema de calidad, y no a los procesos y actividades que se realizan en la empresa y organización. Entonces, el aseguramiento de la calidad consiste en el seguimiento de unas líneas de actuación planificadas y sistemáticas, implantadas dentro del Sistema de Gestión de Calidad de la empresa. Estas acciones deben ser demostrables con el objeto de proporcionar la confianza adecuada, tanto a la propia empresa como a los clientes y proveedores. En consecuencia, para desarrollar e implementar el aseguramiento de la calidad en cualquier organización es necesario que la dirección de la empresa esté plenamente implicada y convencida de que la adopción de este sistema redundará en la buena marcha de la misma, elegir el modelo a implementar y anunciarlo a todos los niveles de la organización además de elegir un responsable para su implementación y mantenimiento forman parte de sus responsabilidades.

Entrando en lo específico del aseguramiento de la calidad en las PYMES ecuatorianas, según datos del último Censo Nacional Económico (2010) del Instituto Nacional de Estadística y Censo (2017) se tiene que el $2.2 \%$ y $5.1 \%$ de pequeñas y medianas empresas, respectivamente, señalan buscar mercados de otros países a la hora de comercializar los bienes y/o servicios que son generados en territorio nacional. Esto debido al acuerdo comercial con la Unión Europea (EU) que entro en vigor el 1 de enero del 2017, donde las 
PYMES podrán acceder a los mercados internacionales o hacer alianzas estratégicas con los países que conforman esta comunidad.

En este sentido, ProEcuador en el año 2016 citado por Tadeo, Vinueza, y Machado (2018) mencionan que Ecuador es el segundo destino de las exportaciones ecuatorianas no petroleras y de los 28 países que componen la UE, 6 de ellos (Alemania, España, Países Bajos, Italia, Francia y Bélgica), concentran el $83.03 \%$ de la oferta ecuatoriana no petrolera hacia la región. El Acuerdo Multipartes entre Ecuador y la Unión Europea, busca, según Ministerio de Comercio Exterior, normar el comercio de bienes y servicios desde y hacia dicho bloque, así como la participación de las partes en licitaciones para la contratación pública, la protección de los derechos de propiedad intelectual, la cooperación en el ámbito del comercio y, en particular disposiciones precisas que establecen un trato especial $y$ diferenciado a favor del Ecuador, en reconocimiento a las asimetrías existentes entre la UE y el Ecuador.

De acuerdo a Andrade y Meza (2017), el Acuerdo Multipartes entre Ecuador y la Unión Europea permite el acceso a mercados más grandes. Las micro, pequeñas y medianas empresas (MiPymes) ecuatorianas podrán incrementar su oferta de exportación si cumplen con los estándares de calidad internacional requeridos. En la UE, el mercado es de aproximadamente 500 millones de habitantes en 28 países, la riqueza cultural y la diversidad de costumbres lo convierten en un mercado muy amplio con consumidores distintos y preferencias distintas, esto representa una oportunidad y una fuerte ventaja para los exportadores del Ecuador que fácilmente pueden incrementar las exportaciones no petroleras hacia este bloque económico puesto que el país tiene como segundo destino más importante de exportaciones no petroleras a la UE y tras la firma del acuerdo podría fácilmente convertirlo tras el paso de los años en su principal socio comercial debido a la ventaja del cero por ciento de arancel.

Otro beneficio de dicho acuerdo según los autores Andrade y Meza (2017) se centra en la transferencia de tecnología ya que para el país este acuerdo plasma un equilibrio entre la apertura comercial con otros aspectos importantes de desarrollo, como la cooperación y transferencia de tecnología. La Unión Europea tiene una industria altamente competitiva a nivel mundial de producción de maquinarias y equipos. El acuerdo comercial con ese bloque permitirá al Ecuador reducir los costos de adquisición del conocimiento tecnológico europeo a través de la importación de bienes de capital necesarios para la industrialización

Este acuerdo conlleva a que las PYMES ecuatorianas entren en un campo de mayor competitividad puesto que las empresas que se encuentran en este mercado registran los mayores números de certificaciones de calidad (ISO) así como también de normas europeas de estandarización para el aseguramiento de la calidad, lo que forja a las PYMES a prepararse al nivel de éstas para poder mantenerse en este ambiente de globalización y estandarización al cual han entrado. 


\section{CONCLUSIONES}

Es de gran notoriedad que las PYMES ecuatorianas ejercen una fuerte influencia sobre el crecimiento económico del país al ser una elevada fuente generadora de empleo, lo que permite contribuir a la mejora social, económica y financiera para combatir los índices de pobreza y generar cambios en las políticas de productividad nacional que permitan conseguir un desarrollo sostenible en el tiempo; garantizando un campo laboral más amplio y de mayor competitividad.

Por su parte, la implementación de sistemas de gestión de calidad como las Normas ISO en las PYMES, permite que las empresas se modernicen y organicen en su estructura interna y de procesos para poder elevar los estándares de calidad en los productos o servicios ofrecidos al analizar $y$ comprender las necesidades, posibles expectativas y satisfaciendo los requerimientos de los clientes; generando a su vez un aumento en la competitividad empresarial a nivel nacional e internacional, dando lugar a un creciente desarrollo de las compañías nacionales y al avance del país en el sector económico y empresarial.

En tal sentido, el aseguramiento de la calidad de las PYMES también ofrece la oportunidad de expandirse en la era de la globalización a mercados nuevos como el europeo tras el Acuerdo de Multipartes con la Unión Europea (UE) para poder comercializar productos, bienes y servicios en este territorio que cumplan con los estándares de calidad establecidos por este mercado, lo que les permitirá ganar mayor respeto, credibilidad y confianza en sus clientes. 


\section{REFERENCIAS}

Andrade, L., \& Meza, A. (2017). Acuerdo comercial entre Ecuador y la Unión Europea caso del sector bananero ecuatoriano. Revista Espacios 38 (58) , 1-5.

Anexia Consultoria. (24 de octubre de 2018). Ventajas de la norma ISO 9001. Recuperado el 31 de Mayo de 2021, de https://consultoria.anexia.es/blog/ ventajas-de-la-norma-iso-9001.

Delgado, D., \& Chávez, G. (abril de 2018). Las Pymes en el Ecuador y sus fuentes de financiamiento. Revista Observatorio de la economía latinoamericana .

Instituto Nacional de Estadística y Censo. (21 de Noviembre de 2017). Censo Nacional Económico 2010. Recuperado el 01 de Junio de 2021, de https://anda.inec.gob.ec/ anda/index.php/catalog/586

ISO 9000. (2005). NORMA INTERNACIONAL Sistemas de gestión de la calidad Fundamentos y vocabulario. Ginebra, Suiza: Secretaría Central de ISO.

Mendoza, W., Loor, T., Hernández, E., \& Hernández, S. (2018). La calidad total como fuente de ventaja competitiva en las pequeñas y medianas empresas (Pymes) del Ecuador. Revista científica de investigación actualización del mundo de las ciencias , 963-984.

Palacio, A., Lanas, J., Mantilla, D., \& Revelo, E. (2018). Utilización de controles, auditorías administrativas y sistemas de gestión de la calidad en las PYMES. Ciencia América 7(1), 1-14.
Palella Stracuzzi, S., \& Martins Pestana, F. (2010). Metodología de la Investigación Cuantitativa. Carcas, Venezuela: FEDUPEL, Fondo Editorial de la Universidad Pedagógica Experimental Libertador.

Peña, M., \& Vega, N. (2018). Estructura de las Pymes en la Economía Ecuatoriana. Revista SurAcadémica 8(1), 30-34.

Pereira, C. (2019). Actualidad de la gestión empresarial en las pymes. Apuntes Contables 24, 1-15.

Rodríguez, P., \& Lesmes, C. (2019). La trascendencia de las PYMES en el mundo de la cultura organizacional. Bogotá: Monografía de la Universidad Santo Tomás.

SECRETARIA NACIONAL DE PLANIFICACION. (2017). Plan Nacional para el buen vivir 2017-2021. Ecuador: Consejo Naconal de Planificación.

Tadeo, R., Vinueza, E., \& Machado, S. (2018). Análisis del cuerdo comercial multipartes entre Ecuador y la Unión Europea y sus posibles impactos en el sector agrícola de la Provincia de Imbabura. Revista Arjé , 431-442.

Terán, P., \& Alvarado, A. (2017). Mejoramiento de la competitividad en empresas PYMES del Ecuador aplicando Lean Six Sigma: estudio de un caso. Gaceta Sansana, 1(7).

Universidad Cooperativa de Colombia. (2018). Sistema de Gestión de la Calidad. 
$\begin{array}{llllllll}R & \text { E } & \text { V } & \text { I } & S & T & A\end{array}$

Public a n d o

I S S N $13390-9304$

Recuperado el 31 de Mayo de 2021, de https://www.ucc.edu.co/sistema-gestionintegral/Paginas/sistema-gestioncalidad.aspx

Universitat Oberta de Catalunya. (12 de Mayo de 2014). Normas ISO en las PYMES. Recuperado el 01 de Junio de 2021, de https://blogs.x.uoc.edu/calidad-iso/ normas-iso-en-las-pymes/ 